\title{
Apatinib induces apoptosis and autophagy via the PI3K/AKT/mTOR and MAPK/ERK signaling pathways in neuroblastoma
}

\author{
XIYING YU ${ }^{1,2 *}$, HONGJUN FAN ${ }^{3 *}$, XINGRAN JIANG ${ }^{4}$, WEI ZHENG ${ }^{1,2}$, \\ YANAN YANG ${ }^{1,2}$, MEI JIN ${ }^{3}$, XIAOLI MA ${ }^{3}$ and WEI JIANG ${ }^{1,2}$ \\ ${ }^{1}$ Department of Etiology and Carcinogenesis and State Key Laboratory of Molecular Oncology; \\ ${ }^{2}$ Beijing Key Laboratory for Carcinogenesis and Cancer Prevention, National Cancer Center/National Clinical Research Center \\ for Cancer/Cancer Hospital, Chinese Academy of Medical Sciences and Peking Union Medical College, Beijing 100021; \\ ${ }^{3}$ Beijing Key Laboratory of Pediatric Hematology Oncology, National Discipline of Pediatrics, Ministry of Education, \\ MOE Key Laboratory of Major Diseases in Children, Hematology Oncology Center, Beijing Children's Hospital, \\ Capital Medical University, National Center for Children's Health, Beijing 100045; ${ }^{4}$ Department of \\ Pathology, Beijing Chaoyang Hospital, Capital Medical University, Beijing 100020, P.R. China
}

Received October 1, 2019; Accepted May 27, 2020

DOI: $10.3892 / \mathrm{ol} .2020 .11913$

\begin{abstract}
The clinical outcome of neuroblastoma (NB) has significantly improved in the last 30 years for patients with localized disease; however, the overall survival (OS) for patients with metastasis remains poor. Apatinib, a selective inhibitor of the vascular endothelial growth factor receptor-2 (VEGFR-2) tyrosine kinase, which was discovered to be highly associated with metastasis, has been reported to exert antitumor effects in numerous types of cancer. However, the effect of apatinib in NB remains relatively unknown. The present study aimed to investigate the antitumor effects of apatinib in NB cells in vitro. The results revealed that apatinib inhibited
\end{abstract}

Correspondence to: Professor Wei Jiang, Department of Etiology and Carcinogenesis and State Key Laboratory of Molecular Oncology, National Cancer Center/National Clinical Research Center for Cancer/Cancer Hospital, Chinese Academy of Medical Sciences and Peking Union Medical College, 17 Panjiayuannanli, Beijing 100021, P.R. China

E-mail: wjiang6138@cicams.ac.cn

Professor Xiaoli Ma, Beijing Key Laboratory of Pediatric Hematology Oncology, National Discipline of Pediatrics, Ministry of Education, MOE Key Laboratory of Major Diseases in Children, Hematology Oncology Center, Beijing Children's Hospital, Capital Medical University, National Center for Children's Health, 56 Nanlishi Road, Beijing 100045, P.R. China

E-mail: mx11123@vip.sina.com

*Contributed equally

Key words: neuroblastoma, apatinib, PI3K/AKT/mTOR pathway, mitogen-activated protein kinase/ERK pathway, apoptosis, autophagy cell viability and colony formation, whilst inducing cell cycle arrest and the apoptosis of NB cells. Additionally, apatinib inhibited the migration and invasion of NB cells, in addition to promoting the autophagy of NB cells. Western blotting demonstrated that the protein expression levels of phosphorylated (p)-AKT, p-mTOR and p-P70S6K, and downstream molecules associated with the cell cycle and apoptosis, such as cyclin D1 and the Bcl-2/Bax ratio of NB cells, were significantly decreased following treatment with apatinib. In addition, western blotting and immunofluorescence assays identified that the expression level of microtubule-associated protein 1A/1B-light chain 3-II, which is expressed in autophagosomes, was upregulated following apatinib treatment. In conclusion, the findings of the present study suggested that apatinib may induce apoptosis and autophagy via the PI3K/AKT/mTOR and mitogen-activated protein kinase/ERK signaling pathways in NB cells. Thus, apatinib may be a potential antitumor agent for the clinical treatment of NB.

\section{Introduction}

Neuroblastoma (NB) is the most common extracranial solid tumor that occurs in children, with $>90 \%$ of patients being diagnosed before the age of 10 years (1). In fact, it is estimated that NB affected 10.72/1,000,000 children $<15$ years each year in the United States between 2001-2009 (2). At the time of diagnosis, $50 \%$ of all NB cases present with metastasis, which are classified as a high-risk clinical phenotype (3). Although the treatment of high-risk NB has significantly improved in the past few decades, the long-term survival rate remains $<50 \%$, which is markedly lower compared with other pediatric oncology diseases (4).

Apatinib is a novel tyrosine kinase inhibitor of the vascular endothelial growth factor receptor-2 (VEGFR2) (5). At the cellular level, apatinib has been reported to selectively block 
the downstream signal transduction of VEGFR2 (6). Apatinib has previously been demonstrated to exhibit promising antitumor effects in various types of tumor in vitro, such as osteosarcoma, anaplastic thyroid cancer, lung cancer and hepatocellular carcinoma (7-10). In addition, in a phase 3 clinical trial, apatinib was identified to significantly improve the median overall and progression-free survival time of advanced gastric cancer, which was refractory to fewer than two lines of prior chemotherapy (11). However, to the best of our knowledge, the antitumor effects of apatinib in NB remain unclear.

The antitumor effects of apatinib reportedly act downstream of the VEGFR2 signaling pathway, such as via $\mathrm{PI} 3 \mathrm{~K} / \mathrm{AKT} / \mathrm{mTOR}, \mathrm{STAT} 3 / \mathrm{Bcl}-2$ or mitogen-activated protein kinase (MAPK)/ERK $(7,12,13)$. In particular, it has been reported that the upregulation of phosphorylated (p)-AKT, p-mTOR and MAPK are recurrent events occurring in NB cell lines, and predict a poor prognosis for patients with NB (14-16).

The present study evaluated the antitumor effects of apatinib and revealed the main signaling pathway affecting the induction of apoptosis and autophagy in NB cells in vitro.

\section{Materials and methods}

Chemicals. Apatinib was obtained from Jiangsu Hengrui Medicine Co., Ltd.. Apatinib (0.5 mg) was dissolved in $1 \mathrm{ml}$ DMSO to a concentration of $1 \mathrm{mM}$ and then diluted with DMEM (cat. no. 10566016; Thermo Fisher Scientific, Inc.) for cell culture.

Cell culture. NB cell lines [BE(2)-M17, SH-SY5Y and IMR-32] were purchased from the American Type Culture Collection. The cells were cultured in DMEM supplemented with $10 \%$ FBS (cat. no. 10100147C; Thermo Fisher Scientific, Inc.) and maintained at $37^{\circ} \mathrm{C}$ with $5 \% \mathrm{CO}_{2}$.

Cell viability, proliferation, cell cycle, apoptosis assays and autophagy observation. Cell viability assays were performed using a Cell Counting Kit-8 (CCK-8; Dojindo Molecular Technologies, Inc.) assay. Briefly, the NB cells were plated at a density of $5 \times 10^{3}$ cells/well into 96 -well plates in triplicate and incubated for $24 \mathrm{~h}$. The effects on harvested cells treated with $20 \mu \mathrm{M}$ near the $\mathrm{IC}_{50}$ concentration of apatinib were too poor to perform the cell cycle assay and other experiments; therefore, lower concentrations of apatinib (5 or $10 \mu \mathrm{M})$ were used in the present study and the results demonstrated an obvious effect of apatinib on NB cells. Subsequently, the cells were cultured with fresh medium containing 0,5 or $10 \mu \mathrm{M}$ apatinib for 24 or $48 \mathrm{~h}$ and the absorbance was subsequently measured at $450 \mathrm{~nm}$ to assess the effect on cell proliferation. The cell cycle and cell apoptosis assays were performed as previously described (17). For autophagy observation, the NB cells were cultured with $10 \mu \mathrm{M}$ apatinib at $37^{\circ} \mathrm{C}$ with $5 \% \mathrm{CO}_{2}$ for $48 \mathrm{~h}$. Then the cellular vacuolization of neuroblastoma cells was visualized via light microscopy (x400 magnification; CKX41; Olympus Corporation, Inc.)

Colony formation assay. BE(2)-M17 and SH-SY5Y cells were treated with 0,5 or $10 \mu \mathrm{M}$ apatinib for $48 \mathrm{~h}$, and then $1 \times 10^{3}$ cells were seeded and cultured in 6-well plates without apatinib. After 14 days of incubation, the cells were fixed with $100 \%$ anhydrous methanol at room temperature for $10 \mathrm{~min}$ and stained with $0.5 \%$ crystal violet at room temperature for 20 min. The colony numbers were observed using a G:BOX Chemi XRQ (Synoptics Ltd., https://www.syngene.com). All experiments were performed in triplicate.

Wound healing and invasion assays. BE(2)-M17 and SH-SY5Y cells were seeded at a density of $5 \times 10^{4}$ cells/well into 6-well plates and cultured until they reached $100 \%$ confluency. Then, pipette tips were used to make a scratch in the monolayer of the cells. Due to the poor adhesion ability of neuroblastoma cells in medium without FBS, the cells were cultured in DMEM supplemented with $2 \%$ FBS and 0,5 or $10 \mu \mathrm{M}$ apatinib. Although the medium with $2 \%$ FBS had little effect on the proliferation of neuroblastoma cells in $24 \mathrm{~h}$, there was a limitation of our wound healing experiment. The wounded area was monitored every $6 \mathrm{~h}$ under a light microscope (x100 magnification; CKX41; Olympus Corporation) and the degree of wound healing induced by cell migration was quantified using Image-Pro Plus software 6.0 (Media Cybernetics, Inc.). The invasion assay was performed as previously described (18). For cell invasion analysis, $1 \times 10^{5}$ cells in serum-free DMEM were plated in the upper membrane of Transwell inserts $(8 \mathrm{~mm})$ coated with $60 \mu \mathrm{l}$ Matrigel (BD Biosciences) in the 24-well plates. High-glucose DMEM containing 20\% FBS was added to the lower wells. Migration was allowed to proceed for $24 \mathrm{~h}$ at $37^{\circ} \mathrm{C}$. Cells that did not migrate through the filters were removed using cotton swabs, and cells that migrated through the inserts were fixed in anhydrous methanol for $10 \mathrm{~min}$ and stained with $0.5 \%$ crystal violet at room temperature for $20 \mathrm{~min}$. The number of migratory cells were observed under a light microscope (x200 magnification; CKX41; Olympus Corporation). All experiments were performed in triplicate.

Bioinformatics analysis. The molecular interactions between the VEGFR2 and PI3K/AKT signaling pathways were analyzed using Search Tool for the Retrieval of Interacting Genes/Proteins (STRING) database (http://string-db.org). In the STRING database, kinase insert domain-containing receptor (KDR) is the official symbol name also known as VEGFR2, and phosphatidylinositol 4,5-bisphosphate 3-kinase catalytic subunit alpha isoform (PIK3CA) is the official symbol name also known as PI3K (19).

Western blotting. Following treatment with apatinib $(0,5$, 10 or $20 \mu \mathrm{M}$ ) at $37^{\circ} \mathrm{C}$ for $48 \mathrm{~h}$ in $5 \% \mathrm{CO}_{2}$, total protein was extracted from BE(2)-M17 and SH-SY5Y cells as previously described (17). In the autophagy assay, the starvation group was cultured without FBS at $37^{\circ} \mathrm{C}$ for $48 \mathrm{~h}$. Total protein was quantified using the BCA Protein Assay kit (cat. no. 23225; Thermo Fisher Scientific, Inc.). Then, $30 \mu \mathrm{g}$ protein/lane was separated on precast $10 \%$ SDS-PAGE gels and subsequently transferred onto polyvinylidene fluoride membranes. The membranes were blocked with $5 \%$ bovine serum albumin dissolved in Tris-buffered saline containing 0.1\% Tween-20 for $30 \mathrm{~min}$ at room temperature followed by incubation with primary antibodies against: Bcl-2 (cat. no. sc-7382; 1:200), Bax (cat. no. sc-7480, 1:200), cyclin D1 (cat. no. sc-8396; 
1:200), p70S6 kinase $\alpha$ (cat. no. sc-230, 1:200), phosphorylated (p)-p70S6 kinase $\alpha^{\text {Ser411 }}$ (cat. no. sc-7983-R, 1:200), mTOR (cat.

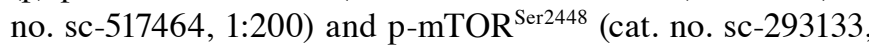
1:200) were purchased from Santa Cruz Biotechnology, Inc., overnight at $4^{\circ} \mathrm{C}$. The primary antibody against $\beta$-actin (cat. no. A1978, 1:10,000) was purchased from Sigma-Aldrich; Merck KGaA. The primary antibodies against AKT (cat. no. 4691, 1:1,000), p-AKT ${ }^{\text {Ser473 }}$ (cat. no. 4058, 1:1,000), ERK (cat. no. 4696, 1:2,000), p-ERK ${ }^{\mathrm{Thr} 202 / \mathrm{Tyr} 204}$ (cat. no. 4376, 1:1,000) and LC3B (cat. no. 3868, 1:1,000) were purchased from Cell Signaling Technology, Inc. The primary antibodies against autophagy related 5 (ATG5; cat. no. 10181-2-AP, 1:1,000) and P62 (cat. no. 18420-1-AP, 1:1,000) were purchased from Proteintech Group, Inc.. Following the primary incubation, membranes were incubated with goat against rabbit IgG-horseradish peroxidase (cat. no. ZB-2301, 1:10,000) and goat against mouse IgG-horseradish peroxidase (cat. no. ZB-2305, 1:10,000; both from Beijing Zhongshan Golden Bridge Biotechnology Co., Ltd.) secondary antibodies for $2 \mathrm{~h}$ at $37^{\circ} \mathrm{C}$. Protein bands were visualized using the Super ECL plus super sensitive luminous liquid (cat. no. P1050; Applygen Technologies, Inc.). The images were captured using the ImageQuant ${ }^{\mathrm{TM}}$ LAS 4000 system (GE Healthcare Life Sciences). The densitometry of the scanned immunoblot bands was analyzed using ImageJ software (version 1.52q; Bharti Airtel Ltd, https://imagej.nih.gov/ij). Each experiment was performed at least three times.

Immunofluorescence. BE(2)-M17 and SH-SY5Y cells were seeded at a density of $1 \times 10^{5}$ cells/well onto glass coverslips in 12 -well plates and treated with 0 or $10 \mu \mathrm{M}$ apatinib for $48 \mathrm{~h}$ at $37^{\circ} \mathrm{C}$ with $5 \% \mathrm{CO}_{2}$. The NB cells were subsequently fixed for immunofluorescence analysis as previously described (20). Following fixation, the cells were penetrated and blocked with PBS containing 10\% FBS and $0.4 \%$ TritonX-100 at room temperature for $30 \mathrm{~min}$. Subsequently, cells were incubated with primary antibodies against LC3B (cat. no. 3868; 1:200; Cell Signaling Technology, Inc.) and Ki-67 (cat. no. 9129; 1:400; Cell Signaling Technology, Inc.) overnight at $4^{\circ} \mathrm{C}$. Following the primary incubation, cells were incubated with rabbit IgG secondary antibody (Alexa Fluor 488, cat. no. ZF-0511; 1:2,000; Beijing Zhongshan Golden Bridge Biotechnology Co., Ltd.) at room temperature for $1 \mathrm{~h}$ and washed three times with PBS containing DAPI (cat. no. D9524; 1:1,000; Sigma-Aldrich; Merck KGaA) at room temperature for $3 \mathrm{~min}$. Cells were observed under a fluorescence microscope (magnification x200; DM2500, Leica Microsystems, Ltd.) or a laser confocal microscope (magnification x400; SP8, Leica Microsystems, Ltd.). Staining intensity was calculated using ImageJ software (version 1.52q; National Institutes of Health).

Statistical analysis. Statistical analysis was performed using SPSS 19.0 software (IBM Corp.). All experiments were performed in triplicate and data are presented as the mean \pm standard deviation. Statistical differences between two groups were determined using unpaired Student's t-test, whereas one-way ANOVA followed by Bonferroni's post hoc test were used for $\geq 2$ groups. $\mathrm{P}<0.05$ was considered to indicate a statistically significant difference.

\section{Results}

Apatinib inhibits cell proliferation and the colony formation of $N B$ cells. To determine the antitumor effects of apatinib on NB cells, the NB cell lines BE(2)-M17, SH-SY5Y and IMR-32 were cultured with different concentrations of apatinib. The CCK- 8 assay revealed that the viability and proliferation of NB cells were significantly inhibited by apatinib in both a dose- and time-dependent manner (Fig. 1A and C). At $48 \mathrm{~h}$, the $\mathrm{IC}_{50}$ of apatinib was $18.74 \mu \mathrm{M}$ for $\mathrm{BE}(2)-\mathrm{M} 17$ cells, $27.37 \mu \mathrm{M}$ for SH-SY5Y cells and $17.09 \mu \mathrm{M}$ for IMR-32 cells (Fig. 1B). Neuroblastoma is frequently associated with the presence of MYCN amplification, a genetic biomarker for poor prognosis (4). The SH-SY5Y cell line without MYCN amplification and BE(2)-M17 cell line with MYCN amplification were selected for further experimentation. Subsequently, BE(2)-M17 and SH-SY5Y cells were treated with apatinib for $48 \mathrm{~h}$ and then seeded into 6-well plates for 14 days. Apatinib significantly decreased the colony formation efficiency of BE(2)-M17 and SH-SY5Y cells (Fig. 1D). In addition, the immunofluorescence assay demonstrated that the expression level of Ki-67 was significantly decreased following apatinib treatment (Fig. 1E). These results indicated that apatinib may inhibit the growth of NB cells in vitro.

Apatinib induces cell cycle arrest and apoptosis of NB cells. To determine the potential mechanism of cell proliferation inhibition, the effects of apatinib on the cell cycle of BE(2)-M17 and SH-SY5Y cells were analyzed using flow cytometry. The cell cycle was significantly arrested at the $G_{0} / G_{1}$ phase in BE(2)-M17 and SH-SY5Y cells following treatment with 5 or $10 \mu \mathrm{M}$ apatinib for $48 \mathrm{~h}$ (Fig. 2A and B). Furthermore, flow cytometric analysis also revealed that apatinib significantly increased the percentage of apoptotic cells in both the BE(2)-M17 and SH-SY5Y cell lines in a concentration-dependent manner (Fig. 2C and D). These findings indicated that apatinib may induce cell cycle arrest and the apoptosis of NB cells.

Apatinib inhibits the migration and invasion of NB cells. To investigate whether apatinib affects the processes of invasion and migration in NB cells, wound healing and Transwell assays were performed. The wound healing assay revealed that apatinib significantly suppressed the migration of BE(2)-M17 and SH-SY5Y cells (Fig. 3A and B). In addition, the invasive abilities of NB cells were significantly reduced following treatment with apatinib for $24 \mathrm{~h}$ (Fig. 3C). These results suggested that apatinib may be a potential anti-metastatic agent for NB.

Apatinib inhibits the PI3K/AKT/mTOR and MAPK/ERK signaling pathways. Bioinformatics analysis revealed molecular interactions between the VEGFR2 and PI3K/AKT signaling pathway (Fig. S1). Western blotting subsequently demonstrated that the expression ratios of $\mathrm{p}-\mathrm{AKT} / \mathrm{AKT}$, p-mTOR/mTOR and p-p70S6K/p70S6K were significantly decreased by apatinib treatment in NB cells (Fig. 4A and B). Since MAPK/ERK is the downstream signaling pathway of VEGFR2 (21), the present study also demonstrated that apatinib inhibited the phosphorylation of ERK in NB cells (Fig. 4C and D). As apatinib induced cell cycle arrest and 

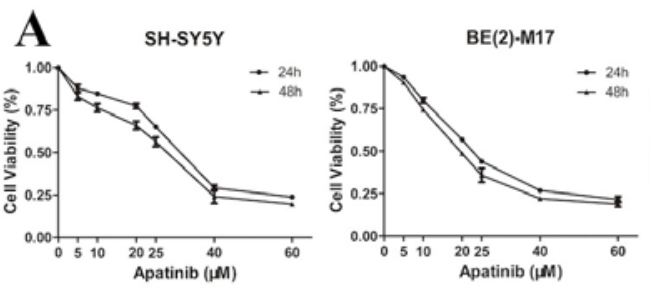

SH-SY5Y

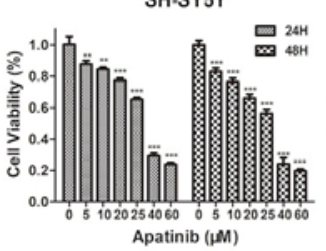

D

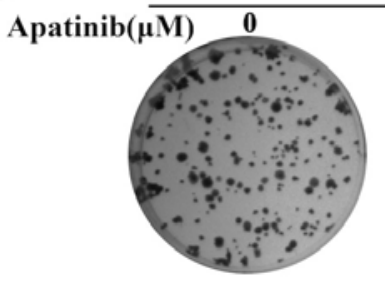

BE(2)-M17

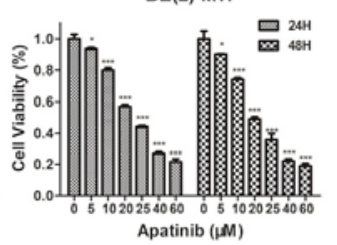

SH-SY5Y

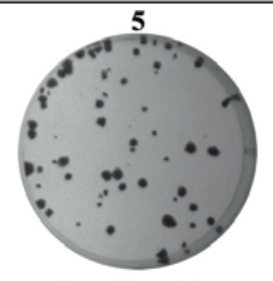

BE(2)-M17

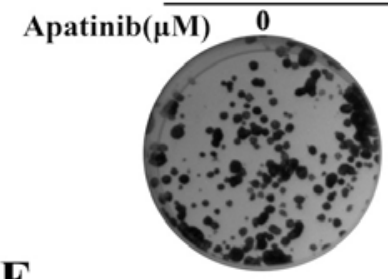

E
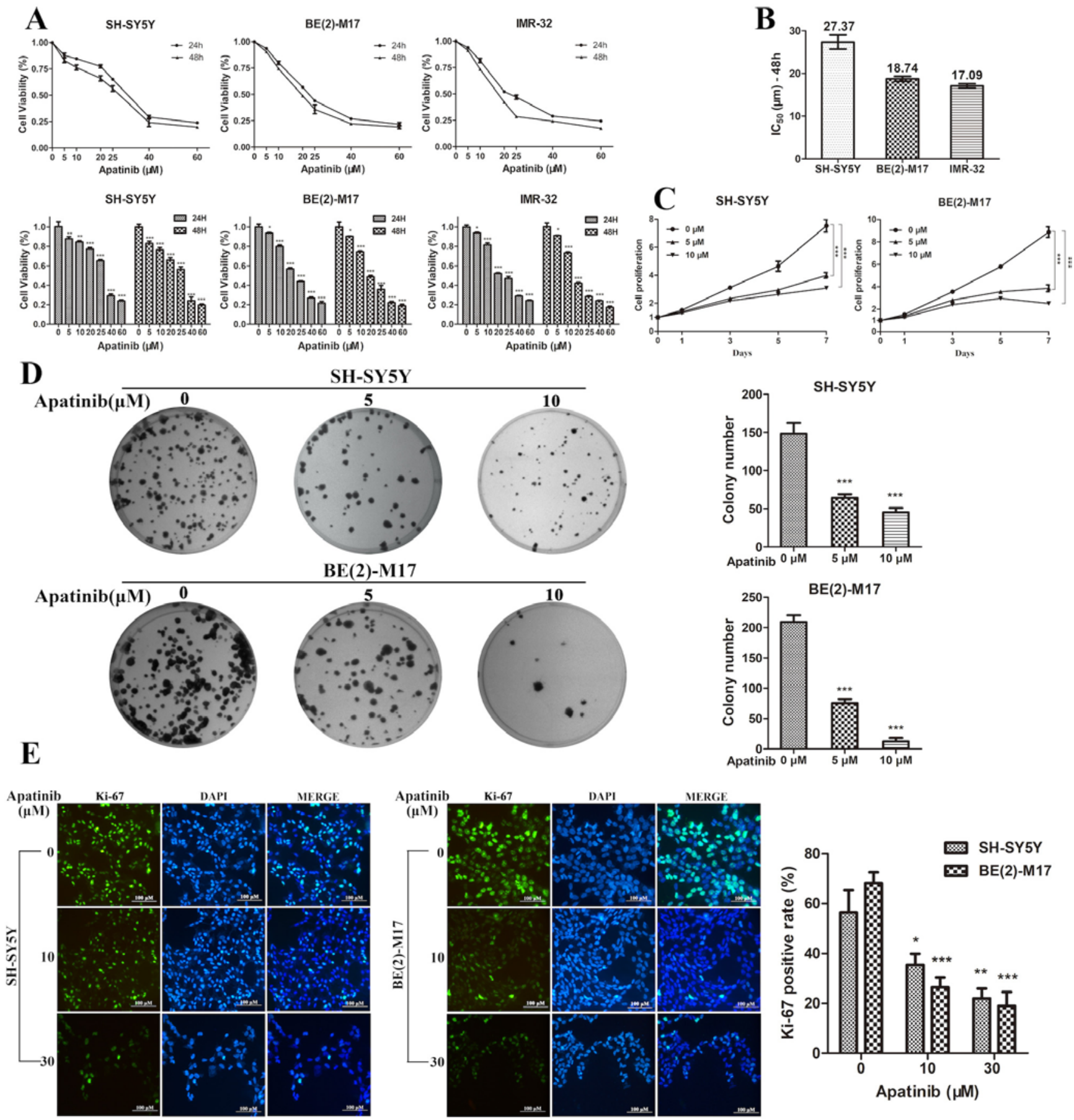

Figure 1. Effects of apatinib on the cell viability, proliferation and colony formation of neuroblastoma cells. (A) Different concentrations of apatinib inhibited the viability of SH-SY5Y, BE(2)-M17 and IMR-32 cells. ${ }^{*} \mathrm{P}<0.05,{ }^{* *} \mathrm{P}<0.01,{ }^{* * *} \mathrm{P}<0.001$ vs. $0 \mu \mathrm{M}$ group. (B) The IC 50 values of apatinib in SH-SY5Y, BE(2)-M17 and IMR-32 cells following the treatment for $48 \mathrm{~h}$. (C) Different concentrations of apatinib inhibited the proliferation rates of SH-SY5Y and BE(2)-M17 cell lines. ${ }^{* * *} \mathrm{P}<0.001$. (D) Apatinib decreased the colony formation rate of SH-SY5Y and BE(2)-M17 cells. ${ }^{* * *} \mathrm{P}<0.001$ vs. $0 \mu \mathrm{M}$ group. (E) The expression of Ki-67 in $\mathrm{SH}-\mathrm{SY} 5 \mathrm{Y}$ and $\mathrm{BE}(2)-\mathrm{M} 17$ cells following treatment with apatinib for $48 \mathrm{~h}$ was detected using immunofluorescence. Scale bar, $100 \mu \mathrm{m}$. $\mathrm{P}<0.05$, ${ }^{* *} \mathrm{P}<0.01$, ${ }^{* * * *} \mathrm{P}<0.001$ vs. $0 \mu \mathrm{M}$ group.

the apoptosis of NB cells, the expression levels of Bcl-2, Bax and cyclin D1 following the treatment with apatinib were also investigated (Fig. 4C and D). The results demonstrated that as the concentration of apatinib increased, the expression of Bcl-2 decreased, while the expression of Bax increased first and then decreased in the SH-SY5Y and BE(2)-M17 cell lines, respectively. It was speculated that the accumulation of apoptotic cells was induced by a high concentration of apatinib, 

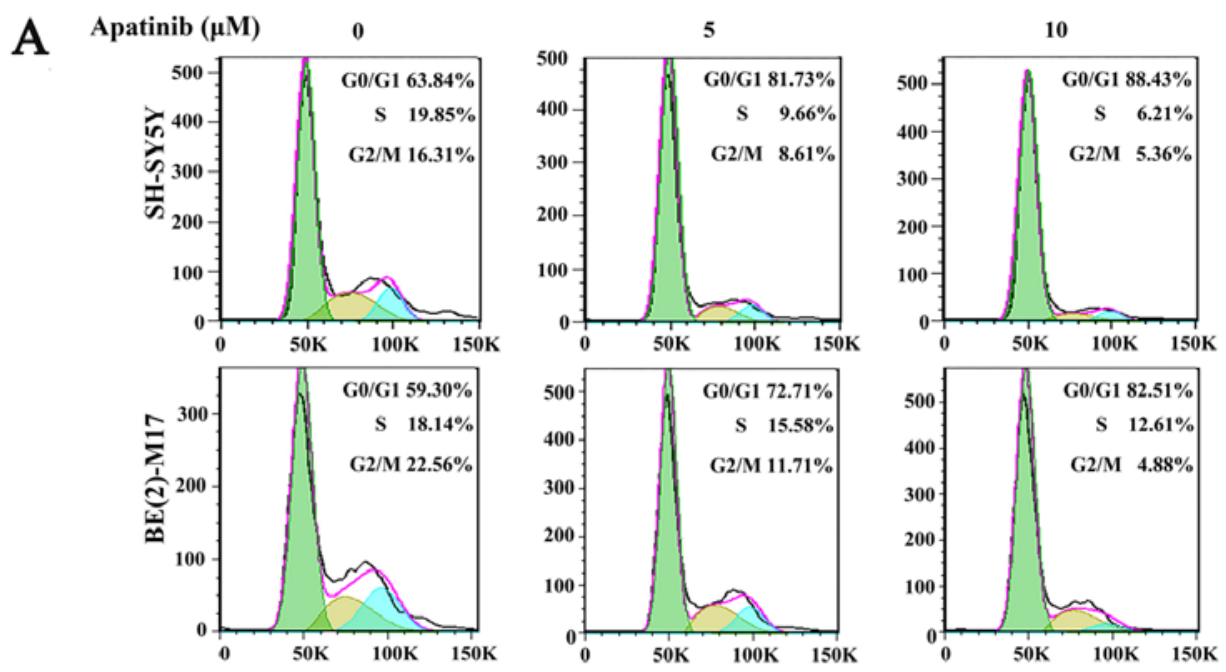

B

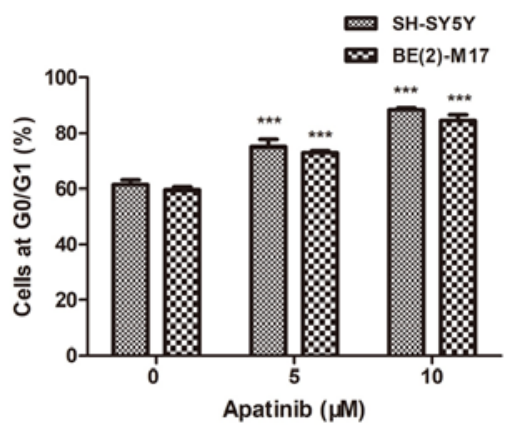

C
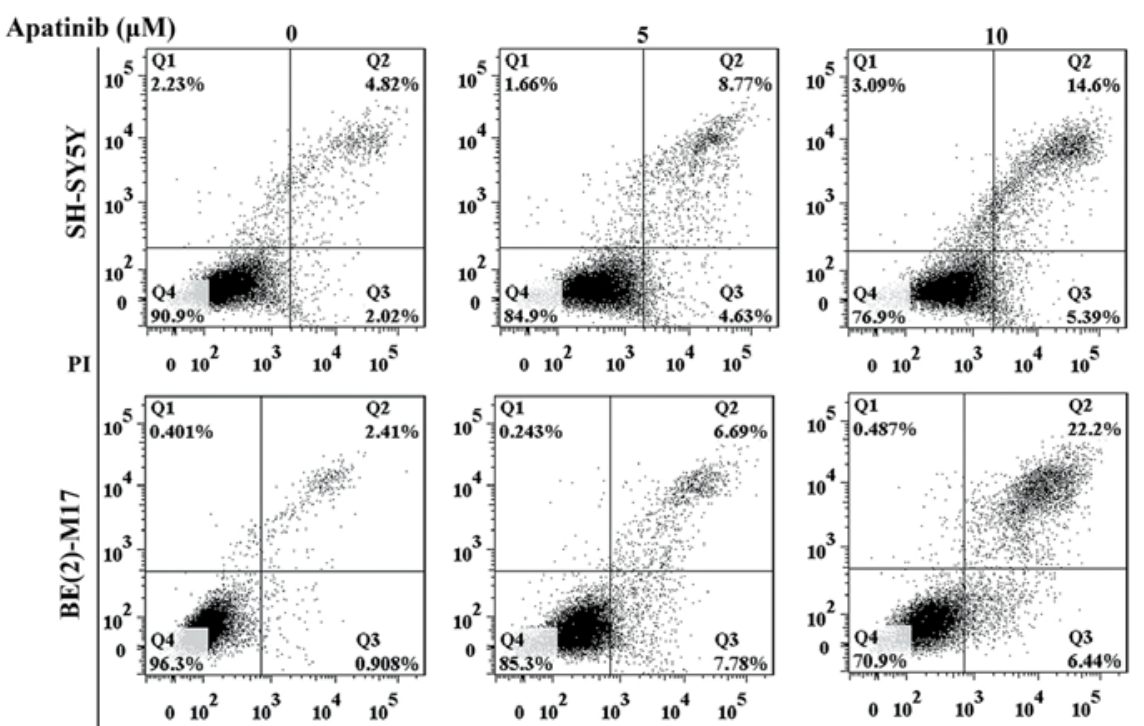

D

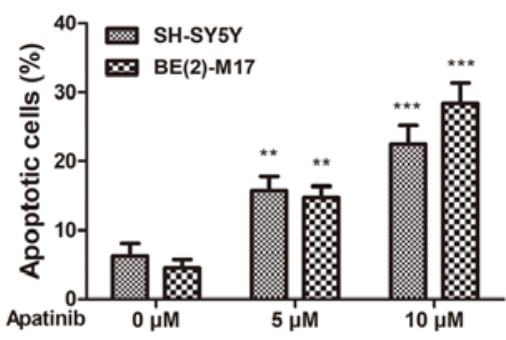

Figure 2. Effects of apatinib on the cell cycle and apoptosis of neuroblastoma cells. (A and B) The cell cycles of SH-SY5Y and BE(2)-M17 cells were arrested at the $\mathrm{G}_{0} / \mathrm{G}_{1}$ phase following treatment with apatinib for $48 \mathrm{~h}$. (C and D) The apoptotic rates of SH-SY5Y and BE(2)-M17 cells following the treatment with apatinib for $48 \mathrm{~h}$. Q2 represents the late apoptotic cells and Q3 represents the early apoptotic cells, and these quadrants were used to quantify the percentages of apoptotic cells. ${ }^{* *} \mathrm{P}<0.01$ and $^{* * *} \mathrm{P}<0.001$ vs. $0 \mu \mathrm{M}$ group. $\mathrm{PI}$, propidium iodide.

Apatinib induces autophagy in NB cells. Furthermore, under the microscope, it was observed that apatinib induced the vacuolization of NB cells (Fig. 5A). Thus, the expression levels of p62, ATG5, LC3-I and LC3-II, which are important proteins involved in the initial stages of autophagy (22), were analyzed. LC3B is the main isoforms of LC3 and was used as an autophagy indicator. The starvation group was used as a positive control of autophagy. The western blotting analysis revealed that following apatinib treatment, the expression levels of p62, ATG5 and LC3B-II/I were significantly increased in NB cells, which was consistent with the starvation group (Fig. 5B). Furthermore, NB cells exhibited a dot fluorescence pattern of LC3B following treatment with apatinib (Fig. 5C). In addition, following apatinib treatment, the ratios of LC3B positive cells in the SH-SY5Y and BE(2)-M17 cell lines significantly increased compared with the control groups. These results suggested that apatinib may induce autophagy in NB cells.

\section{Discussion}

Apatinib, a highly selective tyrosine kinase inhibitor to VEGFR2, has been reported to exert antitumor effects in various types of tumors, such as osteosarcoma, thyroid cancer, cervical cancer and gastric cancer $(7,8,23,24)$. The present study aimed to demonstrate the potential antitumor effects of apatinib in NB cells in vitro. It was identified that apatinib induced apoptosis and autophagy in NB cells, which mainly occurred via the PI3K/AKT/mTOR and MAPK/ERK signaling pathways.

The induction of angiogenesis is a hallmark of cancer (25) and an important step for cancer metastasis (26). It is reported that $\sim 50 \%$ of newly diagnosed patients with NB present with metastasis, usually at the site of the bone or bone marrow (3). Unfortunately, patients with NB that present with bone or bone marrow metastasis have a poor prognosis (27). VEGFR serves an important role in the process of angiogenesis 

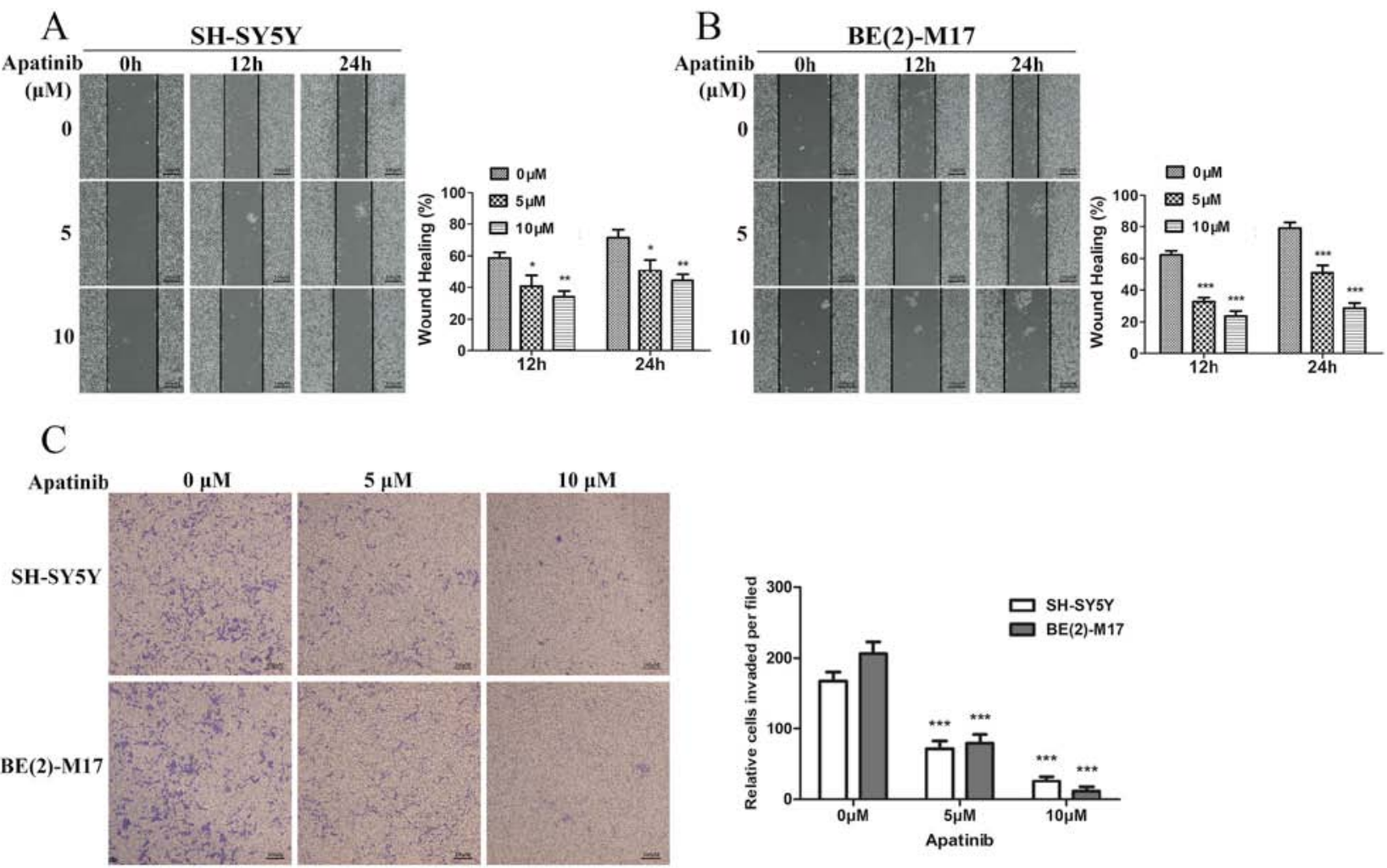

Figure 3. Effects of apatinib on the migration and invasion of NB cells. Apatinib significantly suppressed the migratory ability of (A) SH-SY5Y and (B) $\mathrm{BE}(2)-\mathrm{M} 17$ cells, as determined using a wound healing assay. Scale bar, $100 \mu \mathrm{m} .{ }^{*} \mathrm{P}<0.05,{ }^{* *} \mathrm{P}<0.01,{ }^{* * *} \mathrm{P}<0.001$ vs. $0 \mu \mathrm{M}$ group. (C) Apatinib significantly inhibited the invasive ability of SH-SY5Y and BE(2)-M17 cells after $24 \mathrm{~h}$, as determined using a Transwell assay. Scale bar, $100 \mu \mathrm{m}$. ${ }^{* * * *} \mathrm{P}<0.001 \mathrm{vs} .0 \mu \mathrm{M}$ group.
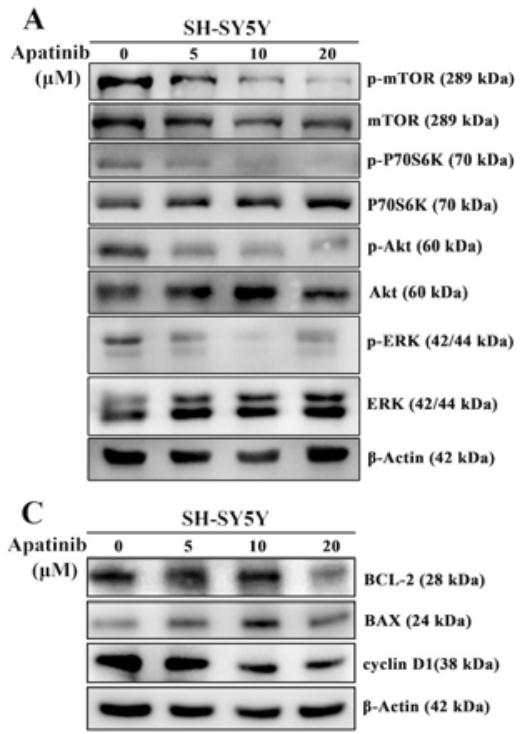
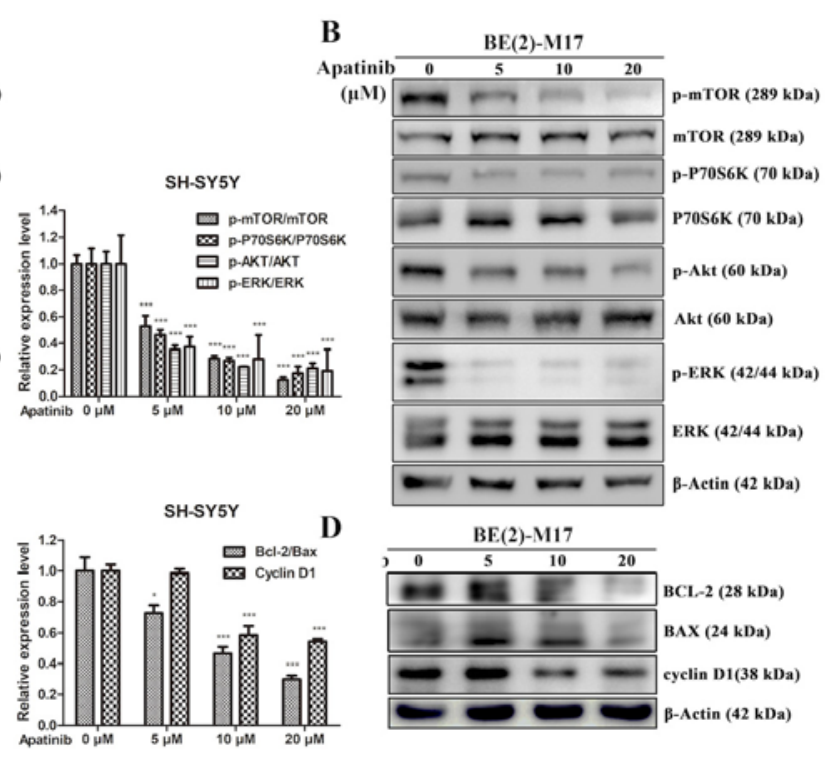

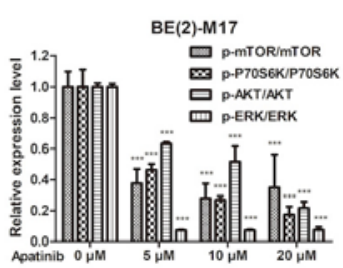

BE(2)-M17

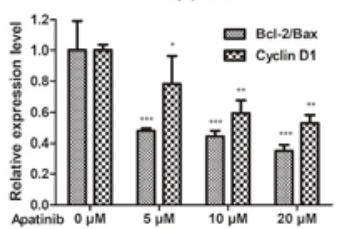

Figure 4. Apatinib inhibits the PI3K/AKT/mTOR and MAPK/ERK signaling pathways. Western blotting also revealed that apatinib downregulated the expression levels of p-AKT, p-mTOR, p-p70S6K and p-ERK in (A) SH-SY5Y and (B) BE(2)-M17 cells after $48 \mathrm{~h} .{ }^{*} \mathrm{P}<0.05,{ }^{* *} \mathrm{P}<0.01,{ }^{* * * *} \mathrm{P}<0.001$ vs. $0 \mu \mathrm{M}$ group. MAPK, mitogen-activated protein kinase; p-, phosphorylated. Western blotting demonstrated that apatinib decreased the Bcl-2/Bax ratio and cyclin D1 expression levels in (C) SH-SY5Y and (D) BE(2)-M17 cells after 48 h. ${ }^{*} \mathrm{P}<0.05,{ }^{* *} \mathrm{P}<0.01,{ }^{* * * *} \mathrm{P}<0.001$ vs. $0 \mu \mathrm{M}$ group.

in cancer (28), and the use of VEGFR tyrosine kinase inhibitors, such as axitinib, sorafenib and sunitinib, has been revealed to effectively inhibit the angiogenesis of NB both in vitro and in vivo (29-31).
It has been reported that apatinib induces apoptosis and autophagy via the VEGFR2/STAT3/Bcl-2 signalling pathway in osteosarcoma and via ER stress in colorectal cancer $(7,32)$. In the present study, apatinib was observed to inhibit cell proliferation, 


\section{$\mathbf{A}$}

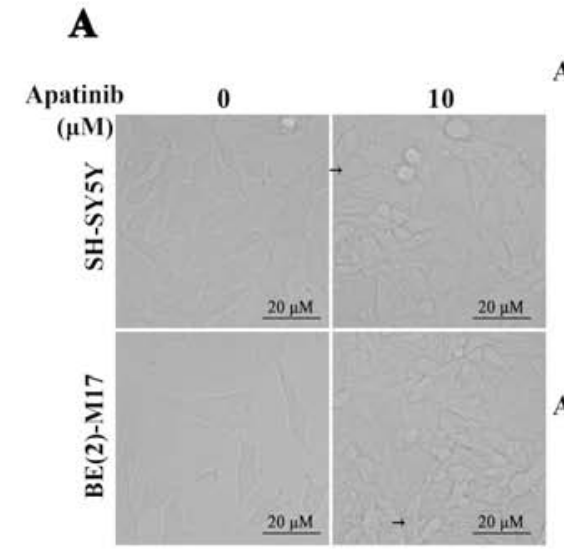

\section{B} \begin{tabular}{llllll}
\cline { 2 - 5 } & $\mathbf{0}$ & 5 & 10 & 20 & starvation
\end{tabular} $(\mu \mathrm{M})$
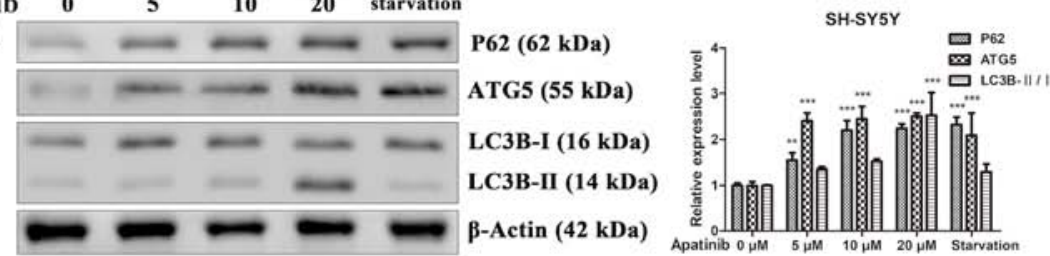

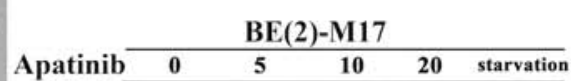

$(\mu \mathrm{M})$

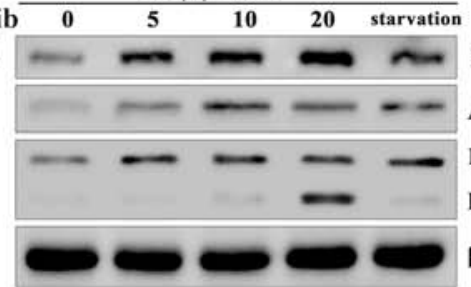

P62 (62 kDa) ATG5 (55 kDa) LC3B-I (16 kDa) LC3B-II (14 kDa) B-Actin (42 kDa)

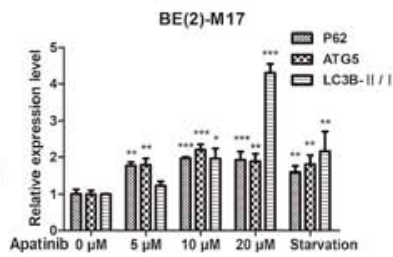

\section{$\mathbf{C}$}
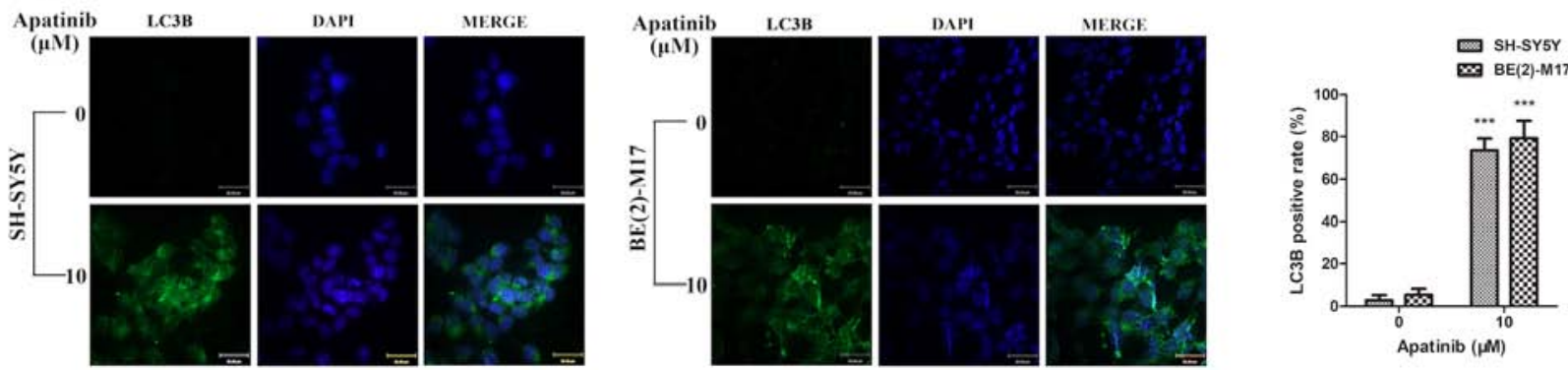

Figure 5. Apatinib treatment induces autophagy in NB cells. (A) Cellular vacuolization induced by $10 \mu \mathrm{M}$ apatinib treatment for $48 \mathrm{~h}$ in SH-SY5Y and BE(2)-M17 cells was visualized using light microscopy. The arrows indicate the vacuoles in NB cells. Scale bar, $20 \mu \mathrm{m}$. (B) Expression levels of P62, ATG5 and LC3B-II/I were significantly downregulated by apatinib in SH-SY5Y and BE(2)-M17 cells. (C) Early autophagosomes stained with LC3B following $10 \mu \mathrm{M}$ apatinib treatment in NB cells for $48 \mathrm{~h}$. Cells were observed by confocal microscopy. Scale bar, $30 \mu \mathrm{m}$. ${ }^{*} \mathrm{P}<0.05,{ }^{* * *} \mathrm{P}<0.01,{ }^{* * * *} \mathrm{P}<0.001 \mathrm{vs} .0 \mu \mathrm{M}$ group. NB, neuroblastoma; ATG5, autophagy related 5; LC3, microtubule-associated protein 1A/1B-light chain 3.

colony formation, and the migration and invasion of NB cells in vitro. The inhibitory effects of apatinib were associated with apoptosis and cell cycle arrest in both a time- and dose-dependent manner. Subsequently, western blotting analysis revealed that the $\mathrm{Bax} / \mathrm{Bcl}-2$ ratio was increased, which is associated with cell apoptosis (33), whilst the expression level of cyclin D1 was downregulated, which is associated with arrest of the cell cycle (34). Furthermore, apatinib inhibited the colony formation, migration and invasion of NB cells. The PI3K/AKT/mTOR andMAPK/ERK signaling pathways regulate numerous cellular processes and physiological functions, including cell proliferation, cell migration, cell metabolism and cell survival $(35,36)$. PI3K/AKT/mTOR and MAPK/ERK act downstream of the VEGFR2 signaling pathway (37). The findings of the present study also demonstrated that apatinib downregulated the phosphorylation of AKT, mTOR, p70S6K and ERK.

Autophagy serves an important role in the progression and treatment of cancer (38). A crucial regulator of autophagy is the nutrient sensor mTOR, which negatively regulates autophagy (38-40). A previous study has revealed that Bcl-2 suppresses autophagy and that inhibiting $\mathrm{Bcl}-2$ promotes the autophagy of cells (41). The presence of LC3 in autophagosomes and the conversion of LC3 to the lower migrating form, LC3-II, are considered to be indicators of autophagy (42). ATG5, which associates with the phagophore, and p62, which targets specific substrates to autophagosomes, may also be used as measurements of the autophagic flux $(38,43)$. In the present study, the expression levels of LC3-II, p62 and ATG5 were significantly upregulated, while the expression levels of Bcl-2 and p-mTOR were significantly inhibited following the treatment with apatinib. These results may further explain the autophagic mechanism of apatinib. However, further in vivo analysis is required to determine whether apatinib can exert antitumor effects and even suppress the metastasis of neuroblastoma by inhibiting angiogenesis via VEGFR2.

In conclusion, to the best of our knowledge, the present study was the first to reveal the antitumor effects of apatinib in NB cells in vitro. The findings suggested that apatinib may inhibit the VEGFR2-dependent PI3K/AKT/mTOR and MAPK/ERK signaling pathways, further to inducing the apoptosis and autophagy of NB cells. These findings may provide a basis for future clinical trials to investigate whether apatinib could be used for the treatment of NB.

\section{Acknowledgements}

Not applicable.

\section{Funding}

The present study was supported by the National Natural Science Foundation of China (grant nos. 81972572 and 
81602138), CAMS Innovation Fund for Medical Sciences (grant no. 2016-I2M-1-001) and the Capital's Funds for Health Improvement and Research (grant no. 2018-2-2095).

\section{Availability of data and materials}

The datasets used and/or analyzed during the present study are available from the corresponding authors upon reasonable request.

\section{Authors' contributions}

$\mathrm{WJ}, \mathrm{MJ}$ and $\mathrm{XM}$ were involved in the conception and design of the study. XY, HF and WZ performed the cellular and molecular experiments. XY, HF, XJ and YY assisted with the statistical analysis. XY and HF were major contributors in drafting the initial manuscript. All authors read and approved the final version of the manuscript.

\section{Ethics approval and consent to participate}

Not applicable.

\section{Patient consent for publication}

Not applicable.

\section{Competing interests}

The authors declare that they have no competing interests.

\section{References}

1. Matthay KK, Maris JM, Schleiermacher G, Nakagawara A, Mackall CL, Diller L and Weiss WA: Neuroblastoma. Nat Rev Dis Primers 2: 16078, 2016.

2. Siegel DA, King J, Tai E, Buchanan N, Ajani UA and Li J: Cancer incidence rates and trends among children and adolescents in the United States, 2001-2009. Pediatrics 134: e945-e955, 2014.

3. Morandi F, Corrias MV and Pistoia V: Evaluation of bone marrow as a metastatic site of human neuroblastoma. Ann N Y Acad Sci 1335: 23-31, 2015.

4. Pinto NR, Applebaum MA, Volchenboum SL, Matthay KK, London WB, Ambros PF, Nakagawara A, Berthold F, Schleiermacher G, Park JR, et al: Advances in risk classification and treatment strategies for neuroblastoma. J Clin Oncol 33: 3008-3017, 2015.

5. Hu L, Sun F, Sun Z, Ni X, Wang J, Wang J, Zhou M, Feng Y, Kong Z, Hua Q and Yu J: Apatinib enhances the radiosensitivity of the esophageal cancer cell line KYSE-150 by inducing apoptosis and cell cycle redistribution. Oncol Lett 17: 1609-1616, 2019.

6. Tian S, Quan H, Xie C, Guo H, Lü F, Xu Y, Li J and Lou L: YN968D1 is a novel and selective inhibitor of vascular endothelial growth factor receptor-2 tyrosine kinase with potent activity in vitro and in vivo. Cancer Sci 102: 1374-1380, 2011.

7. Liu K, Ren T, Huang Y, Sun K, Bao X, Wang S, Zheng B and Guo W: Apatinib promotes autophagy and apoptosis through VEGFR2/STAT3/BCL-2 signaling in osteosarcoma. Cell Death Dis 8: e3015, 2017.

8. Jin Z, Cheng X, Feng H, Kuang J, Yang W, Peng C, Shen B and Qiu W: Apatinib inhibits angiogenesis via suppressing Akt/GSK3ß/ANG signaling pathway in anaplastic thyroid cancer. Cell Physiol Biochem 44: 1471-1484, 2017.

9. Yang C and Qin S: Apatinib targets both tumor and endothelial cells in hepatocellular carcinoma. Cancer Med 7: 4570-4583, 2018.

10. Lin C, Wang S, Xie W, Zheng R, Gan Y and Chang J: Apatinib inhibits cellular invasion and migration by fusion kinase KIF5B-RET via suppressing RET/Src signaling pathway. Oncotarget 7: 59236-59244, 2016.
11. Li J, Qin S, Xu J, Xiong J, Wu C, Bai Y, Liu W, Tong J, Liu Y, $\mathrm{Xu}$ R, et al: Randomized, double-blind, placebo-controlled phase III trial of apatinib in patients with chemotherapy-refractory advanced or metastatic adenocarcinoma of the stomach or gastroesophageal junction. J Clin Oncol 34: 1448-1454, 2016.

12. Feng H, Cheng X, Kuang J, Chen L, Yuen S, Shi M, Liang J, Shen B, Jin Z, Yan J and Qiu W: Apatinib-induced protective autophagy and apoptosis through the AKT-mTOR pathway in anaplastic thyroid cancer. Cell Death Dis 9: 1030, 2018.

13. Huang M, Huang B, Li G and Zeng S: Apatinib affect VEGF-mediated cell proliferation, migration, invasion via blocking VEGFR2/RAF/MEK/ERK and PI3K/AKT pathways in cholangiocarcinoma cell. BMC Gastroenterol 18: 169, 2018.

14. Santo EE, Stroeken P, Sluis PV, Koster J, Versteeg R and Westerhout EM: FOXO3a is a major target of inactivation by PI3K/AKT signaling in aggressive neuroblastoma. Cancer Res 73: 2189-2198, 2013.

15. Mei $H$, Wang $Y$, Lin $Z$ and Tong $Q$ : The mTOR signaling pathway in pediatric neuroblastoma. Pediatr Hematol Oncol 30: 605-615, 2013.

16. Lambertz I, Kumps C, Claeys S, Lindner S, Beckers A, Janssens E, Carter DR, Cazes A, Cheung BB, De Mariano M, et al: Upregulation of MAPK negative feedback regulators and RET in mutant ALK neuroblastoma: Implications for targeted treatment. Clin Cancer Res 21: 3327-3339, 2015.

17. Jiang XR, Yu XY, Fan JH, Guo L, Zhu C, Jiang W and Lu SH: RFT2 is overexpressed in esophageal squamous cell carcinoma and promotes tumorigenesis by sustaining cell proliferation and protecting against cell death. Cancer Lett 353: 78-86, 2014.

18. Xue L, Yu X, Jiang X, Deng X, Mao L, Guo L, Fan J, Fan Q, Wang L and Lu SH: TM4SF1 promotes the self-renewal of esophageal cancer stem-like cells and is regulated by miR-141. Oncotarget 8: 19274-19284, 2017.

19. Wu HB, Yang S, Weng HY, Chen Q, Zhao XL, Fu WJ, Niu Q, Ping YF, Wang JM, Zhang X, et al: Autophagy-induced KDR/VEGFR-2 activation promotes the formation of vasculogenic mimicry by glioma stem cells. Autophagy 13: 1528-1542, 2017.

20. Dong Z, Zhu C, Zhan Q and Jiang W: Cdk phosphorylation licenses Kif4A chromosome localization required for early mitotic progression. J Mol Cell Biol 10: 358-370, 2018.

21. Sun J, Huang W, Yang SF, Zhang XP, Yu Q, Zhang ZQ, Yao J, Li KR, Jiang Q and Cao C: Gail and Gai3mediate VEGF-induced VEGFR2 endocytosis, signaling and angiogenesis. Theranostics 8: 4695-4709, 2018.

22. Larrue C, Saland E, Boutzen H, Vergez F, David M, Joffre C, Hospital MA, Tamburini J, Delabesse E, Manenti S, et al: Proteasome inhibitors induce FLT3-ITD degradation through autophagy in AML cells. Blood 127: 882-892, 2016.

23. Wang Y, Deng M, Chen Q, Li Y, Guo X, Shi P, He L, Xie S, Yu L, Zhang $\mathrm{H}$ and $\mathrm{Xu} \mathrm{B}$ : Apatinib exerts anti-tumor activity to non-Hodgkin lymphoma by inhibition of the Ras pathway. Eur J Pharmacol 843: 145-153, 2019.

24. Qiu H, Li J, Liu Q, Tang M and Wang Y: Apatinib, a novel tyrosine kinase inhibitor, suppresses tumor growth in cervical cancer and synergizes with paclitaxel. Cell Cycle 17: 1235-1244, 2018.

25. Hanahan D and Weinberg RA: Hallmarks of cancer: The next generation. Cell 144: 646-674, 2011.

26. Weilbaecher KN, Guise TA and McCauley LK: Cancer to bone: A fatal attraction. Nat Rev Cancer 11: 411-425, 2011.

27. Viprey VF, Gregory WM, Corrias MV, Tchirkov A, Swerts K, Vicha A, Dallorso S, Brock P, Luksch R, Valteau-Couanet D, et al: Neuroblastoma mRNAs predict outcome in children with stage 4 neuroblastoma: A European HR-NBL1/SIOPEN study. J Clin Oncol 32: 1074-1083, 2014.

28. McTigue M, Murray BW, Chen JH, Deng YL, Solowiej J and Kania RS: Molecular conformations, interactions, and properties associated with drug efficiency and clinical performance among VEGFR TK inhibitors. Proc Natl Acad Sci USA 109: 18281-18289, 2012

29. Rössler J, Monnet Y, Farace F, Opolon P, Daudigeos-Dubus E, Bourredjem A, Vassal G and Geoerger B: The selective VEGFR1-3 inhibitor axitinib (AG-013736) shows antitumor activity in human neuroblastoma xenografts. Int J Cancer 128: 2748-2758, 2011.

30. Kakodkar NC, Peddinti RR, Tian Y, Guerrero LJ, Chlenski A, Yang Q, Salwen HR, Maitland ML and Cohn SL: Sorafenib inhibits neuroblastoma cell proliferation and signaling, blocks angiogenesis, and impairs tumor growth. Pediatr Blood Cancer 59: 642-647, 2012. 
31. Nilsson MB, Zage PE, Zeng L, Xu L, Cascone T, Wu HK, Saigal B, Zweidler-McKay PA and Heymach JV: Multiple receptor tyrosine kinases regulate HIF-1alpha and HIF-2alpha in normoxia and hypoxia in neuroblastoma: Implications for antiangiogenic mechanisms of multikinase inhibitors. Oncogene 29: 2938-2949, 2010.

32. Cheng X, Feng H, Wu H, Jin Z, Shen X, Kuang J, Huo Z, Chen X, Gao H, Ye F, et al: Targeting autophagy enhances apatinib-induced apoptosis via endoplasmic reticulum stress for human colorectal cancer. Cancer Lett 431: 105-114, 2018.

33. Mukhopadhyay S, Panda PK, Sinha N, Das DN and Bhutia SK: Autophagy and apoptosis: Where do they meet? Apoptosis 19: 555-566, 2014.

34. Hydbring P, Malumbres M and Sicinski P: Non-canonical functions of cell cycle cyclins and cyclin-dependent kinases. Nat Rev Mol Cell Biol 17: 280-292, 2016.

35. Thorpe LM, Yuzugullu H and Zhao JJ: PI3K in cancer: Divergent roles of isoforms, modes of activation and therapeutic targeting. Nat Rev Cancer 15: 7-24, 2015

36. Wagner EF and Nebreda AR: Signal integration by JNK and p38 MAPK pathways in cancer development. Nat Rev Cancer 9: 537-549, 2009.

37. Simons M, Gordon E and Claesson-Welsh L: Mechanisms and regulation of endothelial VEGF receptor signalling. Nat Rev Mol Cell Biol 17: 611-625, 2016.
38. Levy JMM, Towers CG and Thorburn A: Targeting autophagy in cancer. Nat Rev Cancer 17: 528-542, 2017.

39. Hansen M, Rubinsztein DC and Walker DW: Autophagy as a promoter of longevity: Insights from model organisms. Nat Rev Mol Cell Biol 19: 579-593, 2018.

40. Zhang G, Wang Z, Du Z and Zhang H: mTOR regulates phase separation of PGL granules to modulate their autophagic degradation. Cell 174: 1492-1506 e22, 2018

41. You L, Wang Z, Li H, Shou J, Jing Z, Xie J, Sui X, Pan H and Han W: The role of STAT3 in autophagy. Autophagy 11: 729-739, 2015.

42. Ohsumi Y: Historical landmarks of autophagy research. Cell Res 24: 9-23, 2014

43. Klionsky DJ, Abdelmohsen K, Abe A, Abedin MJ, Abeliovich $\mathrm{H}$, Acevedo Arozena A, Adachi H, Adams CM, Adams PD, Adeli K, et al: Guidelines for the use and interpretation of assays for monitoring autophagy (3rd edition). Autophagy 12: 1-222, 2016.

This work is licensed under a Creative Commons Attribution-NonCommercial-NoDerivatives 4.0 International (CC BY-NC-ND 4.0) License. 